\title{
Lung ultrasound could reduce X-ray after major lung resection
}

\author{
Dzian $\mathrm{A}^{1}$, Malik $\mathrm{M}^{1}$, Hamada L ${ }^{1}$, Skalicanova $\mathrm{M}^{1}$, Zelenak $\mathrm{K}^{2}$, Stevik $\mathrm{M}^{2}$, Grendar $\mathrm{M}^{3}$ \\ Department of Thoracic Surgery, Jessenius Faculty of Medicine in Martin, Comenius University in Bratislava \\ and Martin University Hospital, Martin, Slovakia. marekmaliksk@gmail.com
}

\begin{abstract}
OBJECTIVES: This study evaluated the role of ultrasound in postoperative care after major lung resection. BACKGROUND: High accuracy of lung ultrasound imaging was proved in various medical fields.

The experience with ultrasound after thoracic surgery is limited.

METHODS: Patients scheduled for major lung resection were consecutively included in a prospective study comparing two modalities of imaging examinations, namely those employing ultrasound and X-ray in the diagnoses of pneumothorax and pleural effusion. Two examinations were performed. One after recovery from anaesthesia, the second before chest tube removal.

RESULTS: Forty-eight patients underwent 87 examinations. X-ray and ultrasound examinations showed substantial and fair agreements for pneumothorax (Cohen's kappa coefficients 0.775 and 0.397 ) and slight and substantial agreements for pleural effusion (Cohen's kappa coefficients 0.036 and 0.611 ). The sensitivity bounds for pneumothorax were $45.5-58.5 \%$ at the first and $29.7-59.4 \%$ at the second examination.

Sensitivity bounds for pleural effusion were $0-86.2 \%$ at the first and $32.6-36.9 \%$ at the second examination. Except for two cases of pneumothorax being missed by X-ray imaging, the rest of mismatches were clinically irrelevant conditions with no impact on clinical decision and patient's outcome.

CONCLUSION: The use of ultrasound can reduce the number of X-ray examinations and thus lower the radiation exposure after major lung resections (Tab. 4, Ref. 30). Text in PDF www.elis.sk KEY WORDS: ultrasound; major lung resection; pneumothorax; pleural effusion, postoperative care.
\end{abstract}

\section{Introduction}

There is a diversity in the chest tube management and monitoring of postoperative pneumothorax (PTX) and pleural effusion (PE) after non-cardiac thoracic surgery (1-3). Despite the X-ray (CXR) disadvantages (2), it remains a standard imaging modality in this field (4). The strategy varies from daily routine employment of CXR to no use of any medical imaging (5).

In various medical specialties, lung ultrasound (LUS) showed excellent results in the diagnostics of chest pathologies. According to evidence-based medicine principles, guidelines were set out for intensive care medicine in respect of LUS diagnostics of almost all acute chest conditions $(6,7)$. LUS was successfully used also in traumatology (8). The experience with LUS in postoperative care after non-cardiac thoracic surgery is limited (9-11) and the results of performed trials did not meet the expectations. The recent trial

${ }^{1}$ Department of Thoracic Surgery, Jessenius Faculty of Medicine in Martin, Comenius University in Bratislava and Martin University Hospital, Martin, Slovakia, ${ }^{2}$ Department of Radiology, Jessenius Faculty of Medicine in Martin, Comenius University in Bratislava and Martin University Hospital, Martin, Slovakia, and ${ }^{3}$ Biomedical Centre Martin, Jessenius Faculty of Medicine in Martin, Comenius University in Bratislava, Martin, Slovakia

Address for correspondence: M. Malik, MD, Department of Thoracic Surgery, Jessenius Faculty of Medicine in Martin, Comenius University in Bratislava and Martin University Hospital, Kollarova 4248/2, SK-036 59 Martin, Slovakia.

Phone: +421.434203415 carried out by Galetin et al showed promising results (12). In this trial, the sensitivity for clinically relevant PTX, i.e., PTX larger than $3 \mathrm{~cm}$ (13), was $100 \%$.

Video-assisted thoracoscopic surgery allows the performance of a whole range of thoracic procedures including the major lung resection to be minimally invasive (14). Guidelines were created to improve the patient outcome after mini-invasive major lung resection (15). Recommendations regarding the use of imaging modalities are non-existent. We wanted to assess whether LUS performed by a thoracic surgeon could reduce the use of CXR and thus lower the use of radiation after major lung resection. To our best knowledge, the role of LUS in detecting PTX and PE in postoperative care after major lung resection alone has not been examined yet. In all studies performed in this field, the major lung resections were just a part of a large mix of procedures such as nonanatomical lung resections or procedures without lung resection.

\section{Materials and methods}

From September 2017 to March 2019, 50 adult patients scheduled for major lung resection at our department were consecutively, non-selectively included in a prospective trial. Two patients after pneumonectomy were excluded. The clinical trial was approved by ethics committee of the University Hospital and by ethics committee of the Faculty of Medicine. Written informed consent was obtained from all participants. The study is in accordance with the World Medical Association Declaration of Helsinki, revised in 2000, Edinburgh. 
871-875

Age, sex, weight, height, body mass index, surgical approach, resected part of the lungs, performance of bronchoplasty, chest tube drainage duration and length of stay in hospital were recorded. LUS was performed in all cases so long as CXR was indicated. Patients underwent two CXR examinations. First CXR was performed after recovery from general anaesthesia at the bedside using a mobile device in a sitting position. The second CXR was performed at the Department of Radiology in an anteroposterior projection before removing the chest tube, couple of hours after its clamping. CXR images were evaluated by skilled radiologists blinded to LUS results. LUS examinations were performed by one of three thoracic surgeons certified in ultrasonography. LUS examiners were blinded to CXR results. Ultrasound examinations were performed at the bedside using SonoScape S2 Portable Ultrasound Machine (SonoScape Medical Corp, Shenzhen, China). LUS examinations were focused on PTX and PE diagnoses. In case of an indeterminate result, the reason for inconclusiveness was recorded. The LUS performance time and interval between LUS and CXR were monitored. LUS results did not influence the decision-making process.

For pneumothorax, the patients were examined in a supine position using a linear probe (5.0-10.0 MHz) Sonoscape L741 (SonoScape Medical Corp, Shenzhen, China). The area between the parasternal and midaxillary lines was examined for lung sliding, B-lines and lung point. The absence of lung sliding, and absence of B-lines combined with the detection of lung point were concluded as PTX. The result was concluded as indeterminate if the lung sliding was missing and the lung point was not found.

A convex probe (2.0-5.0 MHz) Sonoscape C344 (SonoScape Medical Corp, Shenzhen, China) was used for pleural effusion detection. The chest was evaluated in a sitting position, from the anterior axillary line to the paravertebral line. An anechoic or hypoechoic area between defined anatomical borders (lung, diaphragm, chest wall) was recorded as PE.

LUS and CXR results were statistically compared. In cases of this indication, the use of gold standard, the computed tomography, would not be ethical. CXR was used as a reference. It is an imperfect reference, but it is still the standard imaging modality in non-cardiac thoracic surgery. Only the results from the side of the surgery were statistically evaluated. Indeterminate results and examinations where one of the modalities was missing were excluded. Mostly, the second ultrasound examination was missing due to examiners' absence over the weekends. The first and second examination results were compared separately because of different patient characteristics at the time of examinations (patient after general anaesthesia, position, pain, mobility). Sensitivity, specificity, positive and negative predictive values were calculated. The usual computing of these statistical measures does not apply here due to CXR being an imperfect reference. The method of Emerson et al was used (16) to take the imperfection of reference into account. The method leads the bounds on the operating characteristics (OC) of LUS, which depend on the OC of CXR, which should be known here with respect to the gold standard method-computed tomography. For PTX, we used sensitivity and specificity of CXR from the meta-analysis by Ding et al (17). For PE, the CXR sensitivity and specificity from the meta-analysis by Yousefifard et al were used
(18). To evaluate the agreement between LUS and CXR Cohen's kappa coefficients were calculated (19). The analysis of mismatch between CXR and LUS results was performed. For statistical analysis, the R version 3.5.2 (R Foundation for Statistical Computing, Vienna, Austria) and boot library (20) together with an in-house code (21) for computing the bounds of Emerson et al were used.

\section{Results}

Forty-eight patients of whom 29 patients were male (60.4\%) underwent 87 examinations. The average age was 59 years (standard deviation $(\mathrm{SD})=13$; range $22-79)$. The average body mass index was $27.3(\mathrm{SD}=4.6$; range 18.1-39.0). Twenty-seven patients underwent a procedure on the right side $(56.3 \%)$. Nineteen patients $(39 \%)$ underwent posterolateral thoracotomy and 29 patients (60.4\%) had uniportal video-assisted thoracoscopic surgery. Bronchoplasty as part of the procedure, wedge lobectomy and sleeve lobectomy were carried out in $4(8.3 \%), 3$ and 1 patients, respectively. More details are shown in Table 1 . The average duration of chest tube drainage was 5.5 days ( $\mathrm{SD}=2.6$; range $1-11$ days). The average length of hospital stay was 9 days ( $\mathrm{SD}=4.2$; range $3-26$ days).

All 48 patients received the first LUS examination. An average examination took 4.1 minutes ( $\mathrm{SD}=1.4$; range $1.5-9$ minutes). An average interval between LUS and CXR was 83 minutes $(\mathrm{SD}=4$ minutes; range 3-352 minutes).

For PTX, the results of 39 patients $(81.3 \%)$ were statistically compared, while nine LUS results were indeterminate (Tab. 2). The percentage of agreement was $92.3 \%$. Cohen's kappa coeffi-

Tab. 1. Surgical approach and resected lobe.

\begin{tabular}{lccr}
\hline & $\begin{array}{c}\text { Posterolateral } \\
\text { thoracotomy }\end{array}$ & $\begin{array}{c}\text { Uniportal video-assisted } \\
\text { thoracoscopic surgery }\end{array}$ & All \\
\hline Right upper lobectomy & $4^{\mathrm{a}}$ & 9 & 13 \\
Middle lobectomy & $1^{\mathrm{b}}$ & - & 1 \\
Right lower lobectomy & 5 & 5 & 10 \\
Left upper lobectomy & 4 & 5 & 9 \\
Left lower lobectomy & $3^{\mathrm{c}}$ & 9 & 12 \\
Right lower bilobectomy & 2 & 1 & 3 \\
\hline
\end{tabular}

${ }^{\mathrm{a}}$ in one case sleeve lobectomy was performed, ${ }^{\mathrm{b}}$ in one case wedge lobectomy was performed, ${ }^{\mathrm{c}}$ in two cases wedge lobectomy was performed

Tab. 2. Ultrasound results compared to those acquired with X-ray in the first and second examinations for pneumothorax and pleural effusion diagnoses.

\begin{tabular}{llcc}
\hline & & $\begin{array}{c}\text { X-ray } \\
\text { positive }\end{array}$ & $\begin{array}{c}\text { X-ray } \\
\text { negative }\end{array}$ \\
\hline Pneumothorax & Ultrasound positive & 7 & 2 \\
1 ${ }^{\text {st }}$ examination & Ultrasound negative & 1 & 29 \\
& Ultrasound indeterminate & 1 & 8 \\
\hline \multirow{2}{*}{ Pneumothorax } & Ultrasound positive & 4 & 4 \\
$2^{\text {nd }}$ examination & Ultrasound negative & 3 & 22 \\
& Ultrasound indeterminate & 2 & 4 \\
\hline \multirow{2}{*}{ Pleural effusion } & Ultrasound positive & 1 & 6 \\
$1^{\text {st }}$ examination & Ultrasound negative & 4 & 32 \\
& Ultrasound indeterminate & 0 & 5 \\
\hline \multirow{2}{*}{ Pleural effusion } & Ultrasound positive & 11 & 1 \\
$2^{\text {nd }}$ examination & Ultrasound negative & 6 & 19 \\
& Ultrasound indeterminate & 0 & 2 \\
\hline
\end{tabular}


Tab. 3. Reasons of indeterminate ultrasound results. Numbers of missed pathologies compared to X-ray are in parentheses.

\begin{tabular}{lcccc}
\hline & PTX $1^{\text {st }}$ examination & PTX $2^{\text {nd }}$ examination & PE $1^{\text {st }}$ examination & PE $2^{\text {nd }}$ examination \\
\hline Absence of lung sliding and lung point & $9(1)$ & $5(2)$ & - & - \\
Subcutaneous emphysema & - & 1 & - & 2 \\
Wound dressing & - & - & 3 & - \\
Poor echogenicity & - & - & 2 & - \\
\hline
\end{tabular}

Tab. 4. Results of the first and the second ultrasound examinations in the diagnosis of pneumothorax and pleural effusion as compared to $\mathrm{X}$-ray as an imperfect reference while using statistical methods of Emerson et al (16).

\begin{tabular}{|c|c|c|c|c|c|c|c|c|}
\hline & \multicolumn{2}{|c|}{ Sensitivity } & \multicolumn{2}{|c|}{ Specificity } & \multicolumn{2}{|c|}{ PPV } & \multicolumn{2}{|c|}{ NPV } \\
\hline & Min. & Max. & Min. & Max. & Min. & Max. & Min. & Max. \\
\hline Pneumothorax $1^{\text {st }}$ examination & $45.5 \%$ & $58.5 \%$ & $91.5 \%$ & $100 \%$ & $77.8 \%$ & $100 \%$ & $72.1 \%$ & $78.7 \%$ \\
\hline Pneumothorax $2^{\text {nd }}$ examination & $29.7 \%$ & $59.4 \%$ & $79.5 \%$ & $100 \%$ & $50 \%$ & $100 \%$ & $62.2 \%$ & $78.2 \%$ \\
\hline Pleural effusion $1^{\text {st }}$ examination & $0 \%$ & $86.2 \%$ & $82.6 \%$ & $88.4 \%$ & $0 \%$ & $33.1 \%$ & $92.5 \%$ & $99 \%$ \\
\hline Pleural effusion $2^{\text {nd }}$ examination & $32.6 \%$ & $36.9 \%$ & $68.5 \%$ & $100 \%$ & $88.3 \%$ & $100 \%$ & $12.2 \%$ & $17.8 \%$ \\
\hline
\end{tabular}

PPV - positive predictive value, NPV - negative predictive value

cient showed substantial agreement with the value of 0.775 . Two clinically irrelevant cases of PTX with lung point in the second intercostal space in the midclavicular line went undetected on CXR. One case of 11-mm PTX was not recognised on LUS but showed on CXR. There was another one case of PTX being missed by LUS out of nine indeterminate results. In all of them, lung sliding, and B-lines were missing, but the lung point was not found (Tab. 3).

For pleural effusion, the results of 43 patients $(89.6 \%)$ were compared while five results were indeterminate (Tab. 3). Cohen's kappa coefficient showed a slight agreement (0.036), while the percentage of agreement was $76.7 \%$ (Tab. 2). Six cases of PE were missed on CXR, while five of them were below the CXR diagnostic threshold. In four cases, CXR showed small opacities in the costophrenic angle, which was concluded as PE by the radiologist, but LUS clearly ruled out PE in these cases. All mismatch cases were without clinical importance and had no impact on postoperative care.

Thirty-nine patients $(81.3 \%)$ received the second ultrasound examination. An average examination took 4 minutes $(\mathrm{SD}=1.1$ minute; range 2-6.5 minutes). The average interval between LUS and CXR was 169 minutes (SD 117 minutes; range 8-447 minutes).

For PTX, results of 33 patients $(84.6 \%$ ) were compared, while 6 LUS results were indeterminate (Tab. 3). Cohen's kappa coefficient showed a fair agreement with the value of 0.397 , while the percentage of agreement was $78.8 \%$ (Tab. 2). The CXR missed four cases of PTX. Two of these cases were clinically irrelevant. In two cases, the lung point was detected in mid axillary line in intercostal spaces V and VII, respectively. Three cases of PTX went unrecognised on LUS. In these cases, CXR showed PTX of $8 \mathrm{~mm}, 12 \mathrm{~mm}$ and 15 $\mathrm{mm}$, respectively, in the cupula of the pleural cavity. Another two cases of PTX being missed by LUS were due to indeterminate result.

For pleural effusion, two out of 39 second examinations were indeterminate because of subcutaneous emphysema. The percentage of agreement was $81.1 \%$ and Cohen's kappa coefficient showed substantial agreement with the value of 0.611 (Tab. 2). One small PE that showed on LUS went undetected on CXR. In three patients, CXR showed a sharp costophrenic angle. In next two cases, radiologists stated a suspicion for PE, but the result was not clear. In these five cases, LUS clearly ruled out PE. In one patient, LUS did not show pleural effusion described on CXR in the interlobar fis- sure. In all these cases the size of pleural effusion was not clinically important, and the results had no influence on postoperative care.

Minimal and maximal values of sensitivity, specificity, positive and negative predictive values of ultrasound are shown in Table 4 as compared to those of CXR by using the methods of Emerson et al for evaluating diagnostic tests with imperfect reference.

Overall, out of all 87 LUS examinations, 67 (77\%) were exhaustive for both PTX and PE which thus allowed us to make a clinical decision regarding the chest tube removal.

\section{Discussions}

Our study showed substantial and fair agreement for pneumothorax diagnosis and slight and substantial agreements for pleural effusion diagnosis when comparing ultrasound examination performed by a thoracic surgeon with the CXR examination during postoperative care after major lung resection. When compared to the trials dealing with non-cardiac thoracic surgery, the sensitivity bounds for pneumothorax and pleural effusion were not significantly better in this study (Tab. 4). A detailed analysis of mismatch cases showed that except for two cases of pneumothorax detected by LUS and not described by radiologists on CXR, all mismatch cases were those of small-size pneumothorax or minor pleural effusion with no clinical importance or impact on clinical decision. According to these results, the replacement of CXR with LUS performed by a thoracic surgeon as part of chest tube management after non-cardiac thoracic surgery is safe and does not change the clinical decision or patient's outcome.

At the time of designing our study, there was only one published paper, namely Goudie's trial (9), that evaluated ultrasound after non-cardiac thoracic surgery. The sensitivity for PTX diagnosis in the latter study was $21.2 \%$. This trial evaluates together a diverse mix of procedures inclusive of major lung resections, non-anatomical lung resections, and procedures without lung resection. The Goudie's trial had several weaknesses. LUS was performed in a sitting position whereas the supine position used in intensive care and traumatology trials might have brought better results $(6,8)$. The absence of lung sliding, and comet-tail artefacts were concluded as PTX without searching for the lung point 


\section{1-875}

which, according to Lichtenstein, is the most specific ultrasound sign to confirm PTX (22). The absence of lung sliding can be seen in various conditions such as acute respiratory distress syndrome, lung atelectasis, pneumonia, etc (7). Goudie et al mixed the results of both hemithoraxes for statistical comparison.

Chiappetta et al evaluated the exhaustiveness of LUS examination for postoperative complications in 24 patients after a whole spectrum of thoracic procedures (10). As compared to CXR, LUS was exhaustive in $67 \%$ of patients after open procedures and in $85 \%$ of cases after mini-invasive procedures with more detected complications.

Patella et al compared LUS and CXR examinations in evaluation of PTX and lung expansion after chest tube removal (11). Sensitivity and specificity were not calculated while the positive predictive value and negative predictive value were $71 \%$ and $100 \%$, respectively. The results showed that LUS can save $86 \%$ of CXR cases. The absence of lung sliding was concluded as an indication of PTX without searching for the lung point.

Nowadays, Galetin et al are very active in this field. In their first trial in this field (12), 123 patients underwent LUS examination after major lung resection and non-anatomical lung resection. BLUE protocol was used (23). The use of statistical methods of Staquet et al (24) when comparing LUS to CXR as an imperfect reference, resulted in sensitivity and specificity for PTX of $32 \%$ and $85 \%$, respectively. For clinically relevant PTX, i.e., PTX $\geq 3$ $\mathrm{cm}$ according to recent guidelines (13), the ultrasound sensitivity and specificity of pneumothorax detection were $100 \%$ and $82 \%$, respectively. In agreement with our results, no clinically relevant case of pneumothorax escaped the diagnosis. In this trial, the ultrasound results indeterminate for the detection of PTX were marked as the presence of pneumothorax for statistical evaluation. In a newer trial by Galetin et al (25), the results indeterminate for PTX detection were excluded from statistical evaluation, as was the case in our trial too. In the study by Galetin et al, the results on sensitivity and specificity range for pneumothorax in 68 patients $(48 \%$ and $81-100 \%$, respectively) were similar to those achieved in our trial. In their latest work, Galetin et al (26) analysed the impact of various patient conditions on LUS sensitivity of detecting pneumothorax after thoracic surgery. On 340 ultrasound examinations of 208 patients, Galetin et al showed that age, gender, body mass index, smoking status, severity of chronic obstructive pulmonary disease, previous ipsilateral operation or irradiation and thoracotomy did not impair the sensitivity or specificity of LUS in postoperative detection of pneumothorax. This result confirmed the versatility of LUS when used in postoperative care after thoracic surgery.

The use of CXR as an imperfect reference is a weakness of the trials evaluating the role of ultrasound in non-cardiac thoracic surgery. Unlike in intensive care and traumatology trials, here, it would not be ethical to use the gold standard, computed tomography due to enormous radiation. Several statistical methods were described for assessing the OC of a tested modality when the reference test is imperfect $(16,24,27)$. Galetin et al used the methods of Staquet et al which make a simplifying assumption of the conditional independence of the tested and reference methods. The two methods are conditionally independent if, given the disease status, the test results are independent. This assumption is implausible in many applied settings and is known to lead to inaccurate and misleading estimates of OC of the tested method (28). Emerson et al demonstrate that the assumption of the conditional independence may severely restrict the values of the disease prevalence and hence, lead to misleading OC estimates (16). These are the reasons why we do not use the method of Staquet et al. Rather, we rely on the work by Emerson et al where the authors give bounds on the true $\mathrm{OC}$ of the new test as a function of the OC of the imperfect reference test, and data on results of the two tests. The sensitivity of LUS is thus given in the form of an interval with minimal and maximal value, which is done also in case of specificity (Tab. 4). In the method of Emerson et al, the disease prevalence is computed from sensitivity and specificity of the reference method with respect to the gold standard and it also depends on the probability that the reference test detects disease, which can be estimated from the data.

The meta-analysis by Ding et al comparing LUS and CXR in PTX diagnostics showed the superiority of LUS (sensitivity $88 \%$, specificity $99 \%$ ) over CXR (sensitivity $52 \%$, specificity $100 \%$ ) (17). The meta-analysis of Yousefifard et al confirmed the superiority of LUS (sensitivity $94 \%$, specificity $98 \%$ ) as compared to CXR (sensitivity $51 \%$, specificity $91 \%$ ) in diagnostics of PE (18).

Touw et al reflected the superiority of LUS over CXR while they used LUS as a reference to CXR in diagnostics of pulmonary complications in 177 patients after cardiothoracic surgery (29). With excellent inter-observer agreement, LUS, as compared to CXR, showed more complications at an earlier timepoint.

Nooitgedacht et al summarized the evidence of postoperative use of LUS in cardiothoracic and non-cardiac thoracic surgery (30). They suggested to use LUS as a primary imaging modality in thoracic surgery, as well as proposed that the future trials should focus on patient outcome, inter-observer agreement and learning curve.

The absence of inter-observer agreement and learning curve, together with the small size of the cohort of patients are the weaknesses of our trial. High share of indeterminate results, especially for PTX, is another weakness. The most frequent cause of indeterminate result was the absence of lung sliding and absence of B-lines without identifying the lung point in our study (Tab. 4), which made it impossible to rule out PTX in 14 out of all 87 examinations (16.1\%). CXR showed PTX only in 3 of these 14 cases $(21.4 \%)$. CXR showed PTX only in 3 of these 14 cases $(21.4$ $\%)$. The reason for not finding the lung point could be that it was located in the area covered with dressing. Based on this analysis, our methodology was insufficient to rule out PTX. To decrease the number of indeterminate results we suggest adding more ultrasound signs to rule out PTX or adopting a whole standardized methodology, for example BLUE protocol (23) like Galetin et al did. In the study by Galetin et al, the lung pulse was concluded the most useful ultrasound sign to rule out PTX. Also, the evaluation of lung consolidations led to better results (12).

LUS does not expose the patient to radiation. It can be performed at the bedside without moving the patient with chest tube drainage. When LUS is performed by a thoracic surgeon, the examiner has the advantage of knowing the clinical details and the possibility to perform physical examination. LUS can be performed 
repetitively, and it can play an important role in minimizing the costs for health care in patients scheduled for major lung resection.

In conclusion, after detailed mismatch analysis, the results of our study showed the important role of lung ultrasound in decreasing the numbers of $\mathrm{X}$-ray examinations and thus lowering the exposure to radiation after major lung resection because no clinically important pneumothorax or pleural effusion escaped the diagnosis. The replacement of CXR with LUS will not change the clinical decision and patient's outcome. Suggestions for future trials in this field were made, namely greater focus on patient outcome instead of direct comparison of LUS with CXR as an imperfect reference, use of standardized ultrasound methodology like BLUE protocol to avoid indeterminate results, performance of interobserver agreement evaluation and learning curve evaluation.

\section{Learning points}

Chest ultrasound can reduce the number of X-ray examinations in postoperative care after major lung resection.

Chest X-ray is an imperfect reference for pneumothorax and pleural effusion with an impact on acquired ultrasound sensitivity and specificity.

\section{References}

1. Coughlin SM, Emmerton-Coughlin HM, Malthaner R. Management of chest tubes after pulmonary resection: a systematic review and meta-analysis. Can J Surg 2012; 55: 264-270.

2. French DG, Dilena M, Laplante S et al. Optimizing postoperative care protocols in thoracic surgery: best evidence and new technology. J Thorac Dis 2016; 8 (Suppl 1): S3-S11.

3. Zardo P, Busk H, Kutshka I. Chest tube management: state of the art. Curr Opin Anaesthesiol 2015; 28: 45-49.

4. Graham RJ, Meziane MA, Rice TW et al. Postoperative portable chest radiographs: optimum use in thoracic surgery. J Thorac Cardiovasc Surg 1998; 115: 45-50.

5. Mets O, Spronk PE, Binnekade J, Stoker J, de Mol BA, Schultz MJ. Elimination of daily routine chest radiographs does not change on-demand radiography practice in post-cardiothoracic surgery patients. J Thorac Cardiovasc Surg 2007; 134: 139-144.

6. Lichtenstein D, Meziere G. Relevance of lung ultrasound in the diagnosis of acute respiratory failure. The BLUE-protocol. Chest 2008; 134: 117-125.

7. Frankel HL, Kirkpatrick AW, Elbarbary $M$ et al. Guidelines for the appropriate use of bedside general and cardiac ultrasonography in the evaluation of critically ill patients-part I: general ultrasonography. Crit Care Med 2015; 43 (11): 2479-2502.

8. Blaivas M, Lyon M, Duggal S. A prospective comparison of supine chest radiography and bedside ultrasound for the diagnosis of traumatic pneumothorax. Acad Emerg Med 2005; 12: 844-849.

9. Goudie E, Bah I, Khereba M et al. Prospective trial evaluating sonography after thoracic surgery in postoperative care and decision making. Eur J Cardiothorac Surg 2012; 41: 1025-1030.

10. Chiappetta M, Meaci E, Cesario A et al. Postoperative chest ultrasound findings and effectiveness after thoracic surgery: A pilot study. Ultrasound Med Biol 2018; 44 (9): 1960-1967.
11. Patella M, Saporito A, Puligheddu C et al. Lung ultrasound to detect residual pneumothorax after chest drain removal in lung resections. Ann Thorac Surg 2018; 105 (5): 1537-1542.

12. Galetin T, Defosse J, Schieren M et al. Sensitivity of chest ultrasound for postoperative pneumothorax in comparison to chest X-ray after lung resecting surgery. Eur J Cardiothorac Surg 2020; 57 (5): 846-853.

13. Baumann MH, Strange C, Heffner JE et al. Management of spontaneous pneumothorax: an American College of Chest Physicians Delphi consensus statement. Chest 2001; 119: 590-602.

14. Mineo TC, Ambrogi V. A glance at the history of uniportal video-assisted thoracic surgery. J Vis Surg 2017; 3: 157.

15. Batchelor TJP, Rasburn NJ, Abdelnour-Berchtold E et al. Guidelines for enhanced recovery after lung surgery: recommendations of the Enhanced Recovery After Surgery (ERAS (R)) Society and the European Society of Thoracic Surgeons (ESTS). Eur J Cardiothorac Surg 2019; 55: 91-115.

16. Emerson SC, Waikar SS, Fuentes C, Bonventre JV, Betensky RA. Biomarker validation with an imperfect reference: Issues and bounds. Stat Methods Med Res 2018; 27 (10): 2933-2945.

17. Ding W, Shen Y, Yang J, He X, Zhang M. Diagnosis of pneumothorax by radiography and ultrasonography: a meta-analysis. Chest 2011; 140: 859-866.

18. Yousefifard M, Baikpour M, Ghelichkhani $P$ et al. Screening performance characteristic of ultrasonography and radiography in detection of pleural effusion; a meta-analysis. Emerg (Tehran) 2016; 4 (1): 1-10.

19. Landis JR, Koch GG. The measurement of observer agreement for categorical data. Biometrics 1977; 33 (1): 159-174.

20. Canty A, Ripley BD. boot: Bootstrap R (S-Plus) Functions. R package version 1.3-25 2020.

21. https://github.com/grendar/EmersonBounds Web. 31 May 2020.

22. Lichtenstein D, Mezière G, Biderman $P$, Gepner $A$. The lung point: an ultrasound sign specific to pneumothorax. Intensive Care Med 2000; 26: 1434-1440.

23. Lichtenstein DA. BLUE-protocol and FALLS-protocol: two applications of lung ultrasound in the critically ill. Chest 2015; 147: 1659-1670.

24. Staquet M, Rozencweig M, Lee YJ, Muggia FM. Methodology for the assessment of new dichotomous diagnostic tests. J Chronic Dis 1981; 34: 599-610.

25. Galetin T, Schieren M, Marks B, Defosse J, Stoelben E. Sensitivity of lung ultrasound for the detection of pneumothorax one day after pulmonary resection - a prospective observational study. Eur Surg 2021; 53: 23-28.

26. Galetin T, Merres J, Schieren M et al. Most patient conditions do not a priori debilitate the sensitivity of thoracic ultrasound in thoracic surgery - a prospective comparative study. J Cardiothorac Surg 2021; 16 (1): 75.

27. Umemneku Chikere CM, Wilson K, Graziadio S, Vale L, Allen AJ. Diagnostic test evaluation methodology: A systematic review of methods employed to evaluate diagnostic tests in the absence of gold standard - An update. PLoS One. 2019; 14 (10): e0223832.

28. Hui SL, Zhou XH. Evaluation of diagnostic tests without gold standards. Stat Method Med Res 1998; 7: 354-370.

29. Touw HR, Parlevliet KL, Beerepoot $M$ et al. Lung ultrasound compared with chest X-ray in diagnosing postoperative pulmonary complications following cardiothoracic surgery: a prospective observational study. Anaesthesia 2018; 73: 946-954.

30. Nooitgedacht J, Haaksma M, Touw HRW, Tuinman PR. Perioperative care with an ultrasound device is as Michael Jordan with Scotty Pippen: at its best! J Thorac Dis 2018; 10 (12): 6436-6441. 